\title{
Hyperspherical variables analysis of lattice QCD three-quark potentials: skewed Y-string as the mechanism of confinement?
}

\author{
James Leech $^{1,2}$, Milovan Šuvakov ${ }^{3,4}$, V. Dmitrašinović ${ }^{3, a}$ \\ ${ }^{1}$ University of St. Andrews, The Old Burgh School, Abbey Walk, St. Andrews, Fife KY16 9LB, Scotland, UK \\ 2 Present Address: Thales, Reading, UK \\ ${ }^{3}$ Institute of Physics Belgrade, University of Belgrade, Pregrevica 118, Zemun, P.O. Box 57, 11080 Beograd, Serbia \\ ${ }^{4}$ Department of Health Sciences Research, Mayo Clinic, 200 First Street SW, Rochester, MN 55905, USA
}

Received: 26 October 2020 / Accepted: 13 January 2021 / Published online: 24 January 2021

(C) The Author(s) 2021

\begin{abstract}
We have re-analysed the lattice QCD calculations of the 3-quark potentials by: (i) Sakumichi and Suganuma (Phys Rev D 92(3), 034511, 2015); and (ii) Koma and Koma (Phys Rev D 95(9), 094513, 2017) using hyperspherical variables. We find that: (1) the two sets of lattice results have only two common sets of 3-quark geometries: (a) the isosceles, and (b) the right-angled triangles; (2) both sets of results are subject to unaccounted for deviations from smooth curves that are largest near the equilateral triangle geometry and are function of the hyperradius - the deviations being much larger and extending further in the triangle shape space in Sakumichi and Suganuma's than in Koma and Koma's data; (3) the variation of Sakumichi and Suganuma's results brackets, from above and below, the Koma and Koma's ones; the latter will be used as the benchmark; (4) this benchmark result generally passes between the $\mathrm{Y}$ - and the $\Delta$-string predictions, thus excluding both; (5) three pieces of elastic strings joined at a skewed junction, which lies on the Euler line, reproduce such a potential, within the region where the data sets agree, in qualitative agreement with the calculations of colour flux density by Bissey et al. (Phys Rev D 76, 114512, 2007).
\end{abstract}

\section{Introduction}

Soon after the inception of Quantum Chromodynamics (QCD) Mandelstam [1,4], 't Hooft [2] and Nambu [3] suggested the formation of narrow color-electric flux tubes as the mechanism of confinement. This suggestion has since been essentially confirmed by lattice QCD in two-body $(Q \bar{Q})$ systems. But, its extension to three quarks allows two possibilities: the Y-string and the $\Delta$-string, with the topologies of the

M. Šuvakov is on sabbatical leave of absence at 4) Mayo Clinic.

a e-mail: dmitrasin@yahoo.com (corresponding author) corresponding letters. The question of which type of string best describes the three-quark confinement potential in QCD has been open ever since.

In spite of three decades of efforts in lattice QCD, [511], the functional form of the three-heavy-quark potential remains unknown. Even the two most recent calculations $[10,11]$ have drawn incompatible conclusions. It must be emphasized that the analyses of all of the above lattice calculations used only single-variable fitting - three-body potentials depend on three independent variables, however.

A three-variable analysis naturally leaves more lattitude in the interpretation of lattice results. Consequently, the deviations ("error bars") may - and indeed do - turn out larger than in a single-variable analysis. In Refs. $[12,13]$ we re-analysed the lattice data from $[10,11]$ in terms of three hyperspherical variables, and in the present Letter we compare them for the first time. This re-analyses graphically shows how different the chosen geometries were between the two calculations. There are only two (small) subsets of 3-quark geometries that are common to both [10] and [11]: (a) the isosceles, and (b) the right-angled triangles.

Our re-analyses showed that both calculations suffer from significant, unaccounted for deviations ("effective error bars") from smooth potential curves in the same region of triangle shape space: near the equilateral triangle configuration. In this region, the two string potentials, the $\mathrm{Y}$ and the $\Delta$, are indistinguishable. ${ }^{1}$ We do not attempt to explain these (enhanced) deviations here - for these are a matter for the original authors. At any rate, these two are the latest stateof-the-art calculations. The deviations are much smaller and the resulting potential curves smoother in [11] than in [10]. The resulting functional form of [11] is generally within the

\footnotetext{
${ }^{1}$ It is not the errors relative to the difference that are large here, but the absolute errors.
} 
deviations of [10], i.e., [11] curve may be viewed as the converged result, within two (small) subsets of their common 3-quark geometries: (a) isosceles, and (b) right-angled triangles. Here the "converged" data pass between the Y- and the $\Delta$-string predictions, thus excluding both. We re-iterate that the above statement is subject to the above proviso that in certain regions of shape space, both sets of results are subject to unaccounted for deviations from smooth curves that are largest near the equilateral triangle point in shape space.

In a different line of enquiry, Bornyakov et al. [14] calculated and graphically displayed the geometrical distribution of the color-flux density among three static quarks. In the equilateral triangle configuration three flux tubes meet at the unique triangle center, but in asymmetrical triangle configurations Bissey et al. [15] showed that this three-tube junction moves and stays away from the Fermat-Torricelli center required by the Y-string, thus leaving many open questions.

As stated above, we showed that neither the Y-string nor the $\Delta$-string model form acceptable descriptions of the lattice data $[10,11]$. The lattice three-static-quark potential extracted from the small overlap of Refs. [10,11] agrees with the potential resulting from three pieces of elastic string joined together at a skewed Y-junction, which lies on the Euler line, but is displaced from the Fermat-Torricelli center in the direction pointing away from the barycenter of the triangle. This skewed Y-string model is also in qualitative agreement with the results of the lattice calculation of SU(3) color fluxtube configurations connecting three static quarks, or $\mathrm{SU}(3)$ sources, by Bissey et al [15], and thus offers a potential end to this long-standing dilemma.

\section{Lattice QCD data}

The two calculations have several important differences in implementation: Koma and Koma used a $24^{4}$ lattice at $\beta=6.0$ with a lattice spacing $a=0.093 \mathrm{fm}, 221$ threequark geometries and only one gauge configuration, using the "multilevel algorithm" technique and the Polyakov loop; Sakumichi and Suganuma did theirs using the Wilson loop at two $\beta$ values: (a) $\beta=5.8$ on $16^{3} \times 32$ lattice $(a=0.148$ (2) $\mathrm{fm})$ and (b) $\beta=6.0$ on $20^{3} \times 32$ lattice $(a=0.1022(5)$ $\mathrm{fm}$ ), with 1000-2000 gauge configurations, and 101 and 211 three-quark geometries, respectively. The Sakumichi and Suganuma study [10] may be viewed as an update on the Takahashi et al. study [9] made some 13 years earlier: their choices of geometries, and the methods are quite similar, as are the conclusions.

Because of the differing implementations, the studies have different systematic and statistical error bars. The error bars estimated by the original authors have sometimes turned out insufficient to cover the apparent deviations from smooth continuous curves, particularly in the region near the equi- lateral configuration, see Sect. 4.2. That is a matter for the original authors to (re)consider, however; we neglect such troublesome regions of shape space.

Our strategy is to compare the two studies [10] and [11] on a common baseline by (re)expressing both data sets in terms of three-body hyperspherical coordinates. We then compare the results in the regions of common shape-space - where the studies used three-quark triangles of the same shape, though not necessarily of the same size.

We then compare the two sets of extracted data against the predicted Y-string and $\Delta$-string potentials to see if and when, they agree, within their differing error bars, and if they suggest the Y-string or the $\Delta$-string model explanation.

\section{Hyper-spherical coordinates}

In the hyper-spherical coordinate system, a three-quark system is described by the hyper-radius, $R=\sqrt{\frac{1}{3} \sum_{i<j}^{3}\left(\mathbf{r}_{i}-\mathbf{r}_{j}\right)^{2}}$, which is proportional to the root-mean-square distance of the three particles from their geometrical barycenter and thus denotes the size of the system and two hyper-spherical angles, $\alpha=\arccos \left(\frac{2|\rho \times \lambda|}{\rho^{2}+\lambda^{2}}\right)$ and $\phi=\arctan \left(\frac{2 \rho \cdot \lambda}{\rho^{2}-\lambda^{2}}\right)$, or the $(x, y)$ coordinates in the equatorial plane $x=\left(\frac{2 \rho \cdot \lambda}{\rho^{2}+\lambda^{2}}\right)$ and $y=\left(\frac{\rho^{2}-\lambda^{2}}{\rho^{2}+\lambda^{2}}\right)$, which define the shape of the three-quark triangle. Here $\boldsymbol{\rho}=\frac{1}{\sqrt{2}}\left(\mathbf{r}_{1}-\mathbf{r}_{2}\right), \boldsymbol{\lambda}=\frac{1}{\sqrt{6}}\left(\mathbf{r}_{1}+\mathbf{r}_{2}-2 \mathbf{r}_{3}\right)$ are the Jacobi relative vectors.

The hyperradius $R$ scales linearly, $R \rightarrow \lambda R$, with $\lambda$ under dilations/contractions of spatial coordinates $\mathbf{r}_{i} \rightarrow \lambda \mathbf{r}_{i}$ $(i=1,2,3)$ and thus measures the size of the system; the two dimensionless ("shape") hyperangles, or some functions thereof, see appendix A. The scaling symmetry properties reflect only on the hyperradius, whereas the permutation symmetry reflects only on the $\phi$ hyperangular dependence.

Hyper-spherical coordinates have four key advantages over the binary separations $r_{i j}=\sqrt{\left(\mathbf{r}_{i}-\mathbf{r}_{j}\right)^{2}}$ as the threebody variables of choice: Firstly, all of the symmetries of the system are accounted for. Secondly, hyper-spherical coordinates allow simple equations to be written to define the functional form of quark confinement potential, see appendix A. Thirdly, hyper-spherical coordinates allow the size dependence of the quark confinement potential to be separated from its shape dependence because the confining potential is homogenous. Fourth, permutation-adapted hyperspherical coordinates graphically display the dynamical symmetry of the Y-string potential, see Appendix A.

The advantages of hyper-spherical coordinates allow the results from [10] and [11] to be compared, in spite of their differing lattice sizes. The standard Ansatz, [5-11,19-21] for 
(a)

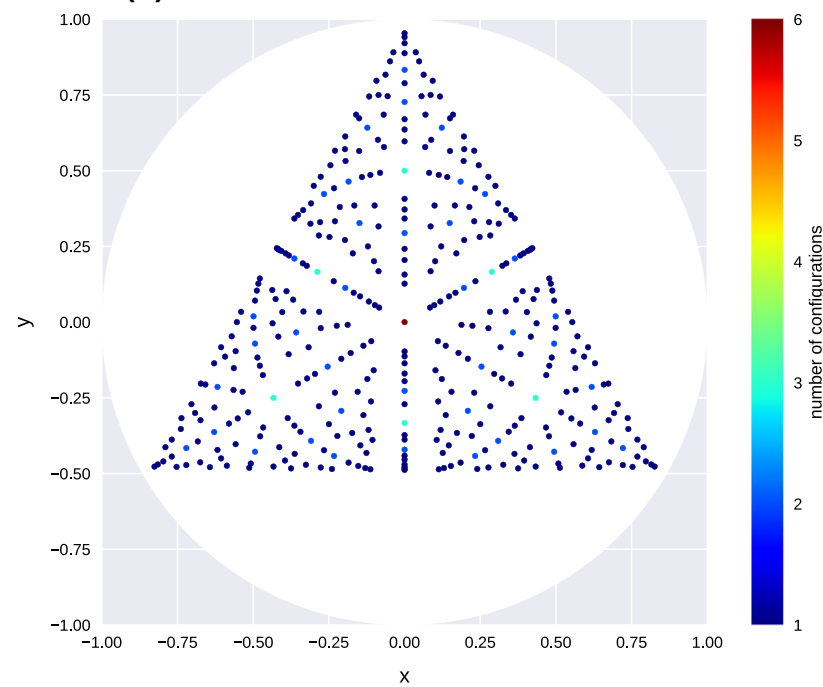

(b)

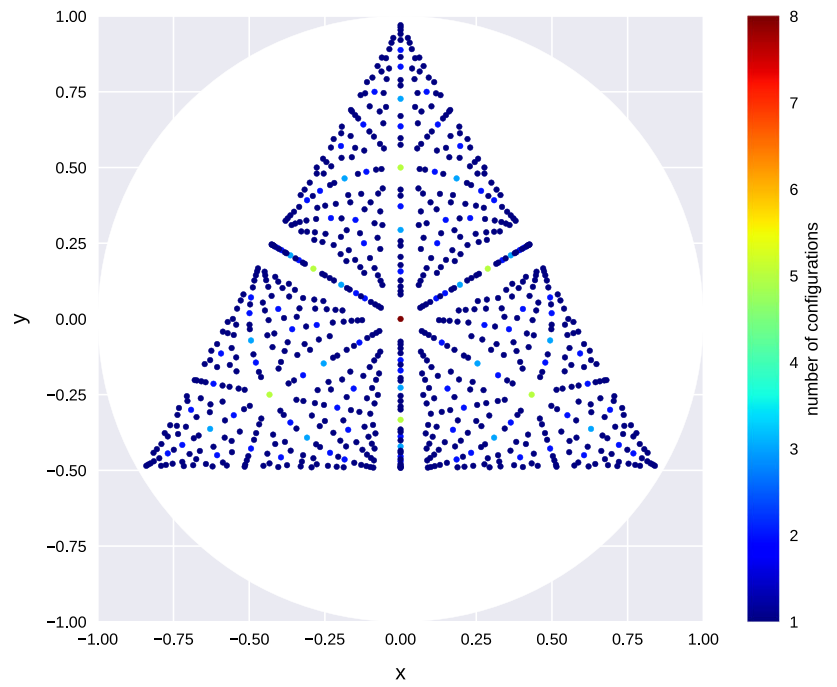

(c)

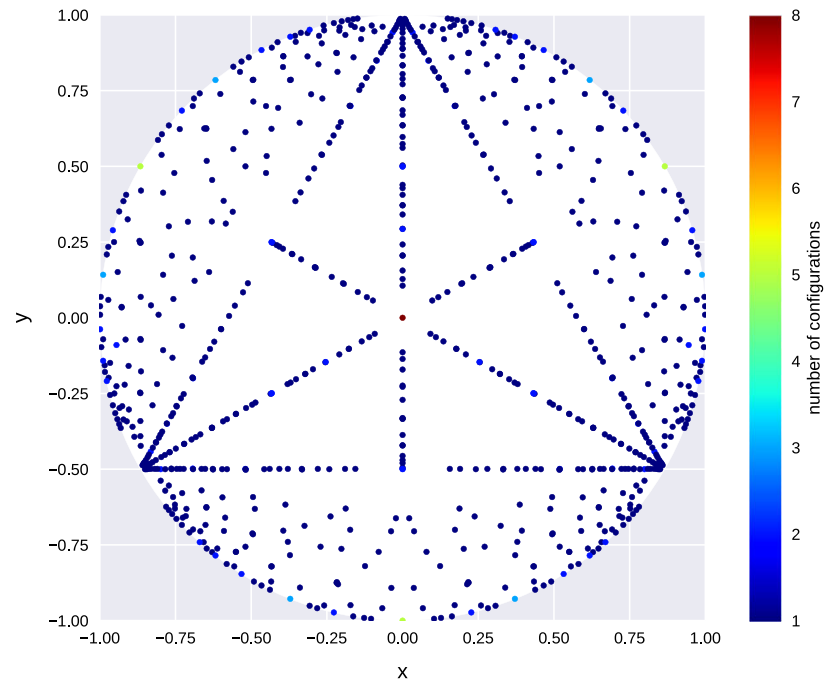

Fig. 1 Distributions of 3q configurations: a Ref. [10] $\beta=5.8$ data; b Ref. [10] $\beta=6.0$ data; and $\mathbf{c}$ Ref. [11] $\beta=6.0$ data. The color code denotes the number of different sizes for an identical configuration. Note the small overlap, and the complementarity of the chosen geometries in Refs. [10] and [11] the three-quark potential $V$ in QCD

$V_{\text {data }}(\alpha, \phi, R)=-\frac{A(\alpha, \phi)}{R}+B(\alpha, \phi) R+C$,

henceforth referred to as the Coulomb+Linear potential, is readily implemented in these variables, and the sizedependence of the confining potential $B(\alpha, \phi) R$ is removed; here $-\frac{A(\alpha, \phi)}{R}$ is the Coulomb part, $B(\alpha, \phi) R$ is the confining part and $C$ is a constant term.

\section{Re-analysis}

\subsection{Method}

At fixed values of shape variables $(\alpha, \phi)$, the functions $A(\alpha, \phi), B(\alpha, \phi)$ and $C(\alpha, \phi)$ are (unknown) constants. In order to determine their values we have fitted the equilateral triangle data. With these constants fixed at one point in the shape-space, we can subtract the well-known Coulomb $V_{\text {Coul }}^{f i t}$ and constant $C_{f i t}$ term in all of shape-space (see appendix B), and be left with the confining potential $V^{*}(x, y) R=V_{\text {data }}-$ $V_{\text {Coul }}^{f i t}-C_{\text {fit }}$.

The distributions of Sakumichi and Suganuma's, as well as of Koma and Koma's three-quark geometries, represented as points in the shape-space disc, are shown in Fig. 1. Note the complementarity of the two sets, and the small overlap regions - two mutually orthogonal straight lines in one elementary "pizza slice" cell - between the two sets of chosen geometries.

The two data sets have been defined in terms of a common hyper-spherical coordinate system and a subset of points has been selected where the two data sets have geometric overlap. Results of Refs. [10] and [11] can now be compared to each other and to the Y-string and $\Delta$-string predictions.

\subsection{Results}

We showed in Ref. [12] that Koma and Koma's [11] lattice results yielded a continuous, generally smooth functional dependence along these two (orthogonal) lines in the shapespace disc. In Ref. [13] we subjected Sakumichi and Suganuma's lattice data [10] to the same analysis as Koma and Koma's [11] in Ref. [12], with somewhat less convincing conclusions: the deviations from a unique, smooth curve are larger than in [11]. Nevertheless, the data in Ref. [10] show a marked improvement over the data in Ref. [9] in terms of reduced deviations. $^{2}$

\footnotetext{
2 We analysed the Takahashi et al. [9] data at the same time as the Koma and Koma's [11] one, but did not publish the results, as the deviations from a continuous curve were too large. We talked about these findings at the 2018 NFQCD meeting at Kyoto [22].
} 
The hyperangular dependences of Sakumichi and Suganuma's [10] and Komas' [11] confinement potentials are shown in Figs. 2 and 3 next to each other, so as to facilitate comparison. The first impression is unfavourable: Sakumichi and Suganuma's isosceles data show large deviations from a smooth curve between $-0.4 \leq y \leq 0.3$ at $\beta=5.8$ (Fig. 2a), and between $-0.4 \leq y \leq 0.7$ at $\beta=6.0$ (Fig. 2b). A correspondingly large deviation in Koma and Koma's isosceles data appears only below $y \leq-0.4$ (Fig. 2c), however.

The right-angled triangle data of Ref. [10], on the other hand, shows similar deviations (only) in the $-0.3 \leq x \leq 0.3$ at $\beta=5.8$ (Fig. 3a), and between $-0.7 \leq x \leq 0.7$ at $\beta=6.0$ (Fig. 3b). A corresponding deviation in Ref. [11] right-triangle data appears only above $|x| \geq 0.75$ (Fig. 3c). Note that in all cases the deviations of Ref. [10] data bracket those of Ref. [11].

One may therefore view Ref. [11] as having superior accuracy to that of Ref. [10]. ${ }^{3}$ Consequently, we shall use Ref. [11] as the benchmark result.

The discerning reader will also notice the hyper-radial dependence of the above bounds in Figs. 2, 3. Our interactive web site [24] allows the interested reader to change the value(s) of the hyperradius $R$, and thus select the data to be observed through filters of one's own choice.

Note that the benchmark data consistently fall between the $\Delta$-string prediction (upper, blue) and the Y-string prediction (lower, green) in the aforementioned region. It ought to be clear that neither the Y-nor the $\Delta$-string can adequately describe the present lattice data.

\section{Interpretation: skewed junction Y-string}

This unexpected result calls for an interpretation in terms of an elastic string model. We define an infinite class of skewed Y-string potentials:

$V_{\text {sk.Y }}=\sigma_{\text {sk.Y }} \sum_{i=1}^{3}\left|\mathbf{x}_{i}-\mathbf{x}_{0}(\alpha)\right|$

whereby three pieces of elastic string are joined at a junction, $\mathbf{x}_{0}(\alpha)$, defined as

$\mathbf{x}_{0}(\alpha)=\mathbf{x}_{0}^{\text {F.T. }}+\alpha \Delta \mathbf{x}_{0}$

Here $\alpha \in(-\infty,+\infty)$ is a free parameter such that the barycenter $\mathbf{x}_{0}^{\mathrm{CM}}=\mathbf{x}_{0}(\alpha=-1)$ corresponds to $\alpha=-1$ :, and the Fermat-Torricelli center $\mathbf{x}_{0}^{\text {F.T. }}=\mathbf{x}_{0}(\alpha=0)$ corresponds

\footnotetext{
${ }^{3}$ Such large deviations did not propagate into the single-variable analysis completed by the original authors. We do not wish to speculate about possible explanations of this fact, but it should be clear that this region appears to require at least a re-analysis of error bars.
}

(a)
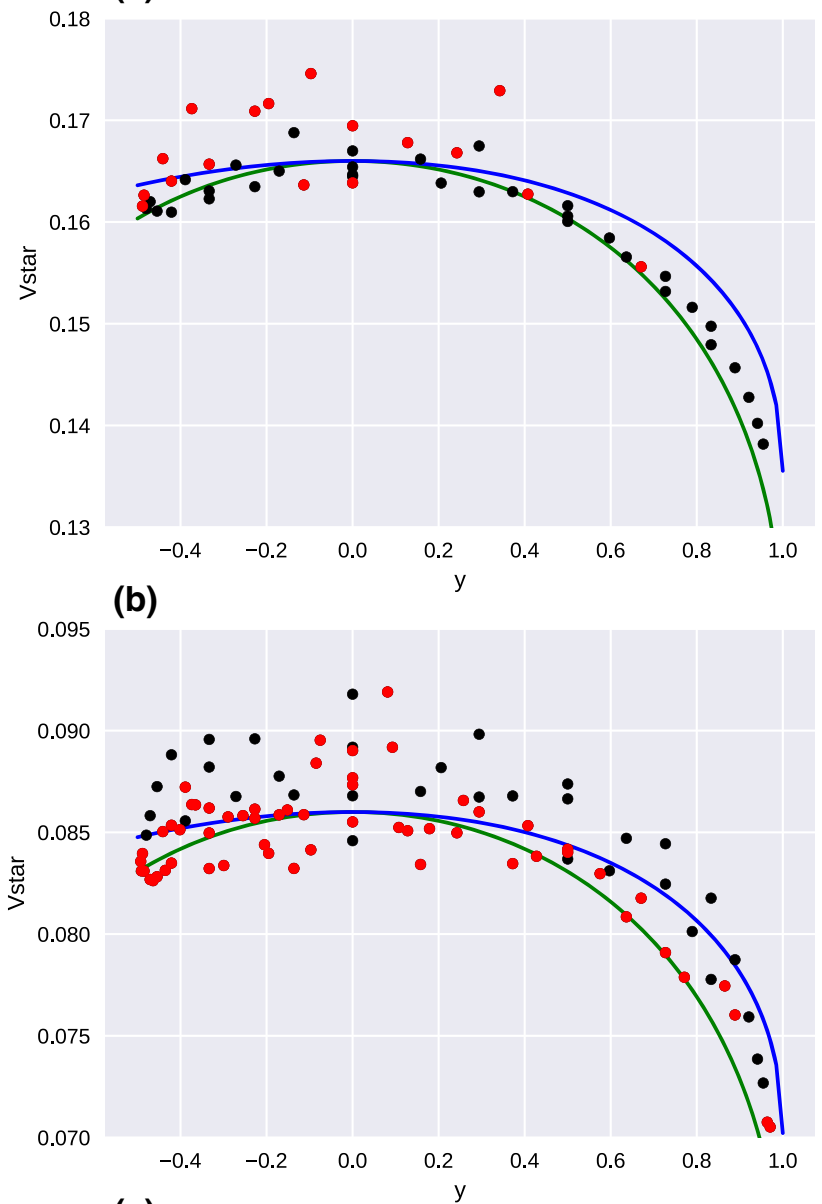

(c)

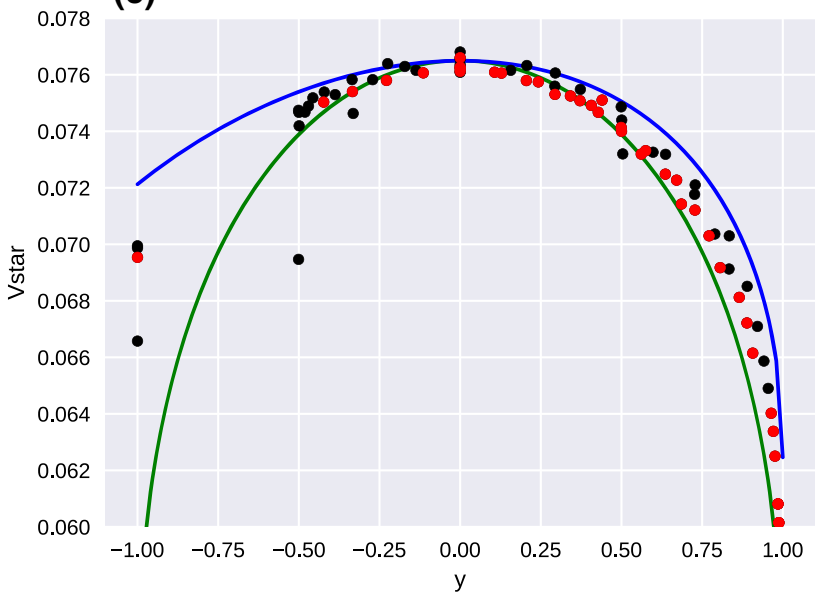

Fig. 2 Extracted values of the hyperangular part of the confining potential $V^{*}(x, y)=1 / R\left[V_{\text {data }}-V_{\text {Coul }}^{f i t}-C_{f i t}\right]$ for isosceles triangles $(x=0.5): \mathbf{a} \beta=5.8$, Ref. [10]; b $\beta=6.0$, Ref. [10]; c $\beta=6.0$, Ref. [11]. The black points correspond to all values of size $R$, whereas the red ones correspond to sizes $R \geq 7$. The blue lines represent the $\Delta$-string prediction, the green lines the Y-string 
(a)

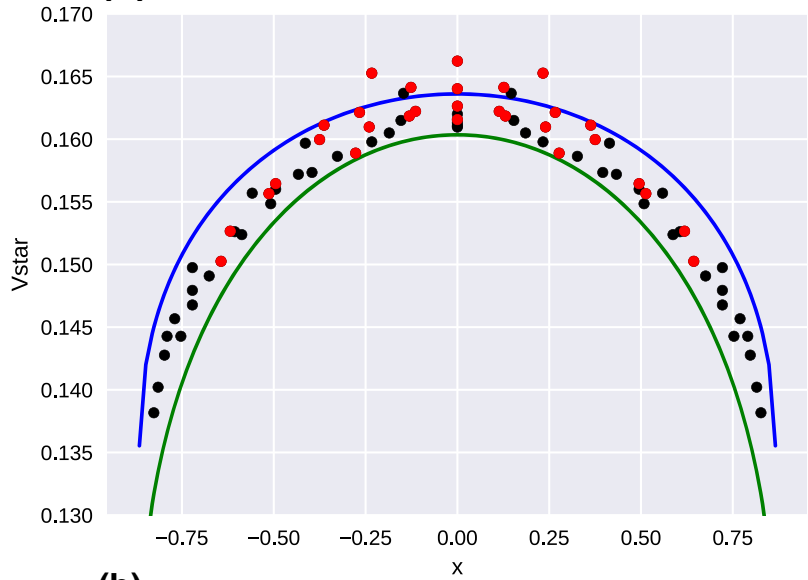

(b)

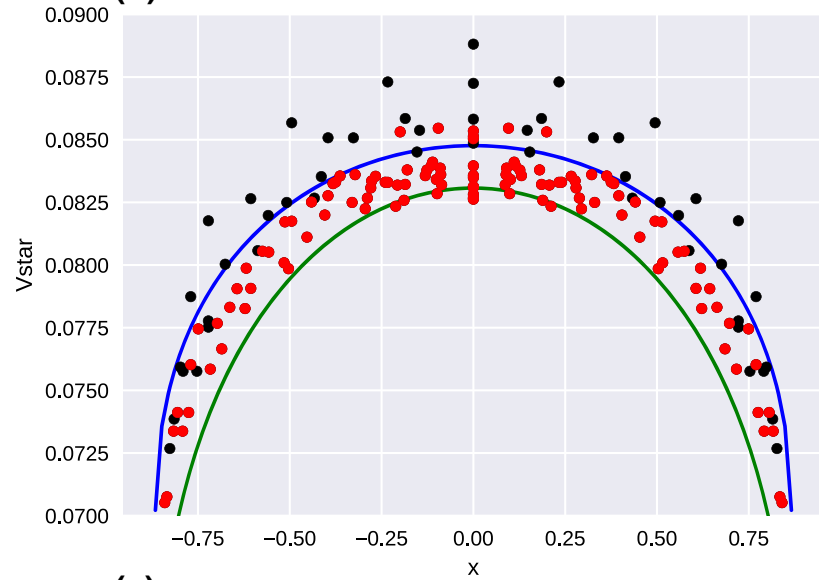

(c)

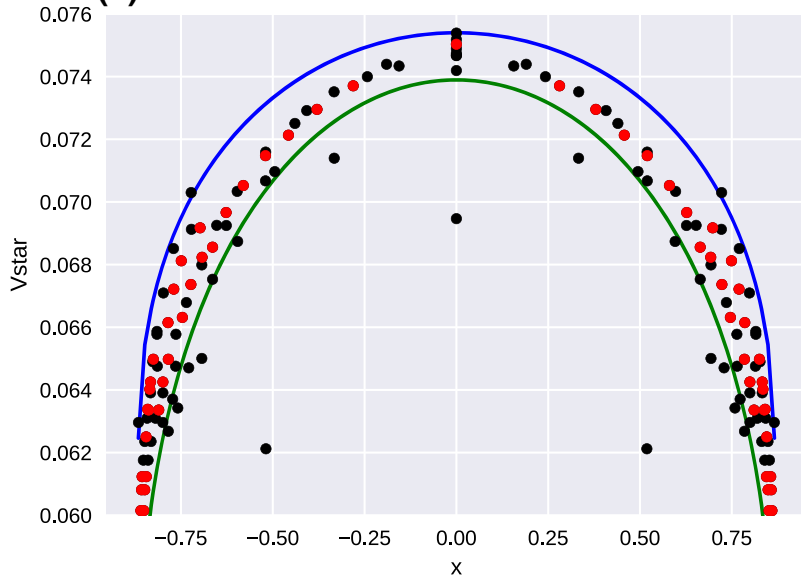

Fig. 3 Extracted values of the hyperangular part of the confining potential $V^{*}(x, y)=1 / R\left[V_{\text {data }}-V_{\text {Coul }}^{f i t}-C_{f i t}\right]$ for right triangles $(y=0)$ : a $\beta=5.8$, Ref. [10]; b $\beta=6.0$, Ref. [10]; c $\beta=6.0$, Ref. [11]. The black points correspond to all values of size $R$, whereas the red ones correspond to sizes $R \geq 7$. The blue lines represent the $\Delta$-string prediction, the green lines the Y-string to $\alpha=0$. Thus the junction lies on the Euler line ${ }^{4}$ defined by the vector $\Delta \mathbf{x}_{0}$

$$
\begin{aligned}
\Delta \mathbf{x}_{0} & =\mathbf{x}_{0}^{\mathrm{F} . \mathrm{T}}-\mathbf{x}_{0}^{\mathrm{CM}} \\
& =\frac{1}{2 l_{Y}^{2}} \sqrt{\frac{3}{2}}\left(\mathbf{V}+\frac{\mathbf{W} \times \mathbf{A}}{|\boldsymbol{\rho} \times \boldsymbol{\lambda}|}\right)
\end{aligned}
$$

where

$$
\begin{aligned}
\mathbf{V} & =\lambda\left(\lambda^{2}-\rho^{2}\right)-2 \rho(\rho \cdot \lambda) \\
\mathbf{A} & =\rho\left(\lambda^{2}-\rho^{2}\right)+2 \lambda(\rho \cdot \lambda) \\
\mathbf{W} & =\rho \times \lambda \\
l_{Y}^{2} & =\frac{3}{2}\left(\rho^{2}+\lambda^{2}+2|\rho \times \lambda|\right)
\end{aligned}
$$

The junction determined by the lattice data is displaced from the Fermat-Torricelli point of the triangle in the direction away from the barycenter, i.e., at positive $\alpha>0$. Any point on the Euler line may be used as a junction of three strings, within a subset of the shape-space disc. This region of applicability of a string potential is determined by the position of the center at which the strings have their junction, and how the position of the center changes with the triangle shape. Of course, in an equilateral triangle all centers coincide. As a triangle shape becomes more obtuse, some of its centers move outside the triangle. When a center moves outside the boundary of the triangle, it stops being acceptable as a junction of three strings. The exit point on the boundary inherits the property of being the junction.

We find that such a string model potential can fit the data in Figs. 2 and 3 , i.e., the lattice results of both [10] and [11], though the spread of the data does not, as yet, allow a more precise determination than $\alpha \simeq 0.5 \pm 0.2$.

The direction of the displacement of the junction from the Fermat-Torricelli center is well established, however, as being opposite to the barycenter. This leads to a reduction of the value of the critical angle, down from $120^{\circ}$, which is sufficient to show that this elastic string model is in qualitative agreement with the results of the lattice calculation of SU(3) color flux-tubes connecting three quarks by Bissey et al. [15], who found flux-tubes in the shape of letters $\mathrm{L}$ and T, i.e., with a critical angle of $90^{\circ}$.

\section{Discussion}

The displacement of the three-string junction from the Fermat-Torricelli point is perhaps the least expected fea-

\footnotetext{
4 The Fermat-Torricelli center of a triangle is one of many - the barycenter, the inscribed center, the circumscribed center being but a few examples - all of which lie on the Euler line. As each such center is defined in a permutation-symmetric way the resulting potential Eq. (2) is also permutation symmetric.
} 
ture of three-quark confinement. Neither Bissey et al. [15], who observed it first, nor anyone thereafter has offered an explanation of the T- and L-letter-shaped flux tubes.

To be fair, de Forcrand and Jahn [17] suggested a theoretical scenario wherein a transition from the $\Delta$-string, holding at shorter distances, to the Y-string holding at longer distances would take place at separations of around $0.8 \mathrm{fm}$. Putting aside, for the time being, theoretical arguments against this scenario advanced in Ref. [16], see also Appendix A, we note that there is no evidence for such a transition taking place in the data shown in Fig. 5, nor in any of the results in Refs. $[10,11]$.

Of course, our conclusions are only as good as the data they are based on, which left a number of things to be desired, so we can only re-iterate that the deviations from continuous curves must be ironed out. Therefore, all further checks, including refutations, corroborations and refinements by future lattice QCD studies will be welcome. A straightforward check would have to contain both sets of geometries (isosceles and right-angled) used so far, at as many different hyper-radii as possible, whereas a refinement would include new type(s) of geometries, again at least at four different values of hyper-radius.

Our result, if correct, has consequences for three-quark spectroscopy and the confinement potential for multiquark systems:

1. in baryon spectroscopy the three-quark force leaves a clear signature in the second odd-parity, and higher shells of baryon resonances, and even there only in a few select states [25-27]. However, these three-quark force effects can easily be confused with relativistic effects. Therefore the shifted junction is not likely to be observed soon in heavy baryon spectroscopy.

2. The displacement of the three-string junction from the Fermat-Torricelli point would dramatically influence the binding energies and confinement properties of multiquark systems. This is because in systems of four or more quarks the Y-string is replaced by a Steiner tree [28], which should be distorted due to the skewness of the junction.

Our results open new questions: firstly, what is the precise position of the string junction for three-quarks at zero temperature? Secondly, what happens to this junction at nonzero temperatures? Thirdly, what are the Casimir scaling properties of the confining 3-body potential? How does the three-body potential depend on the color SU(3) multiplet to which the three bodies belong? It has been suggested [29] that the color SU(3) dependence should be the symmetric structure constants $d^{a b c}$, but that implies its vanishing for self-conjugate multiplets, such as the octet, which would be a dramatic effect.

Acknowledgements We thank Yoshiaki and Miho Koma, and Naoyuki Sakumichi and Hideo Suganuma for kindly sharing their published and unpublished data, respectively. V.D. thanks Y. and M. Koma for illuminating discussions, as well as for their kind hospitality at Numazu College, and H. Suganuma for alerting him to his work. This work was begun in the summer of 2017 when J.L. was visiting IPB on an IAESTE student exchange program. We thank Igor Salom for serving as liaison with the Belgrade office of IAESTE. The work of M. Š and V. D. was partially supported by the Serbian Ministry of Education, Science and Technological Development (MESTD) under Grants No. OI 171037 and III 41011, and partially by IPB through grant by MESTD Republic of Serbia.

Data Availability Statement This manuscript has associated data in a data repository [Authors' comment: See the web site https://suvakov. github.io/3q/ for interactive analysis and https://github.com/suvakov/ $3 q$ for the original data.]

Open Access This article is licensed under a Creative Commons Attribution 4.0 International License, which permits use, sharing, adaptation, distribution and reproduction in any medium or format, as long as you give appropriate credit to the original author(s) and the source, provide a link to the Creative Commons licence, and indicate if changes were made. The images or other third party material in this article are included in the article's Creative Commons licence, unless indicated otherwise in a credit line to the material. If material is not included in the article's Creative Commons licence and your intended use is not permitted by statutory regulation or exceeds the permitted use, you will need to obtain permission directly from the copyright holder. To view a copy of this licence, visit http://creativecomm ons.org/licenses/by/4.0/.

Funded by SCOAP ${ }^{3}$.

Appendix A: Y- and $\Delta$-string potentials in the isosceles and right-angled configurations

The string, Coulomb and the CM-string potentials are defined in terms of $x$ and $y$ as below:

$$
\begin{aligned}
& V_{\Delta}(x, y) \\
& \quad=\sigma_{\Delta}\left(r_{12}(x, y)+r_{23}(x, y)+r_{13}(x, y)\right) \\
& V_{Y}(x, y) \\
& \quad=\sigma_{Y} \sqrt{\frac{3}{2}\left(1+\sqrt{\left|1-\left(x^{2}+y^{2}\right)\right|}\right)} \\
& V_{C M}(x, y) \\
& =\sigma_{1 b}\left(r_{1}(x, y)+r_{2}(x, y)+r_{3}(x, y)\right) \\
& V_{\text {Coulomb }}(x, y) \\
& =K\left(\frac{1}{r_{12}(x, y)}+\frac{1}{r_{23}(x, y)}+\frac{1}{r_{13}(x, y)}\right)
\end{aligned}
$$

where

$r_{23}(x, y)$ 
(a)

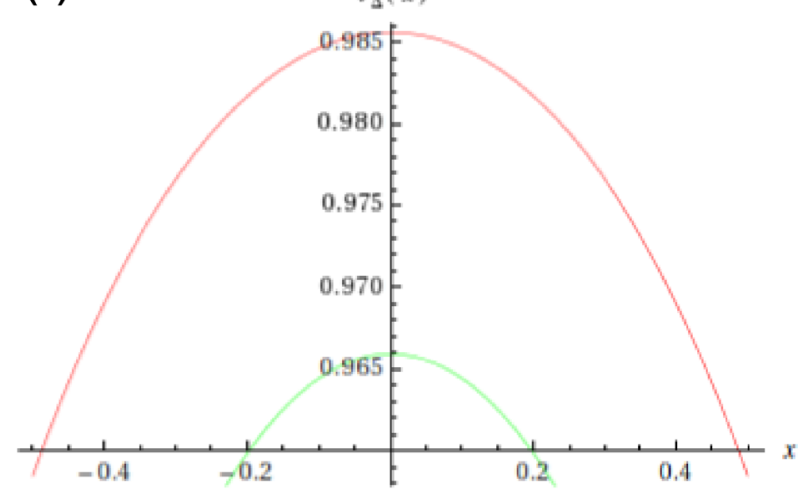

(b)

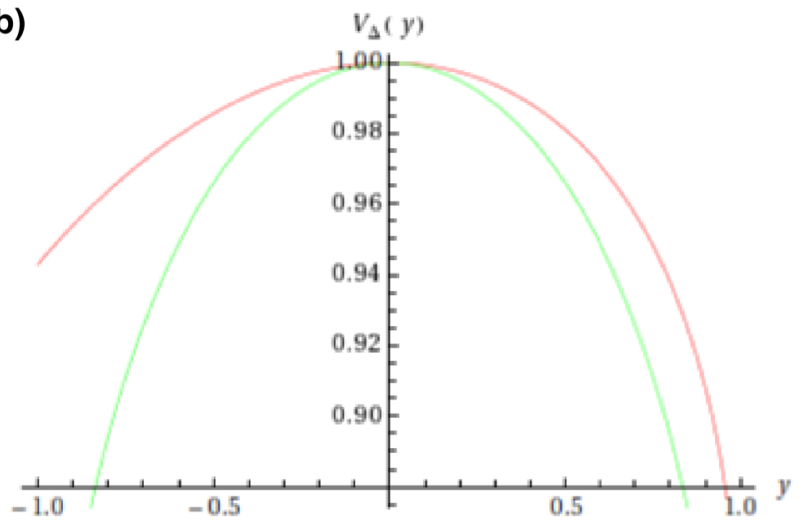

Fig. 4 The 1.h.s. panel a the $\Delta$ (red) and Y-string (green) potentials in the right-angled triangle configurations. r.h.s. panel b The $\Delta$ (red) and Y-string (green) potentials in the isosceles triangle configurations

$$
\begin{aligned}
& =R \sqrt{1+\left(\sqrt{x^{2}+y^{2}}\right) \sin \left(\frac{\pi}{6}-\arctan \left(\frac{y}{x}\right)\right)} \\
& =R \sqrt{1+\frac{x-\sqrt{3} y}{2}} \\
& r_{13}(x, y) \\
& =R \sqrt{1+\left(\sqrt{x^{2}+y^{2}}\right) \sin \left(\frac{\pi}{6}+\arctan \left(\frac{y}{x}\right)\right)} \\
& =R \sqrt{1+\frac{x+\sqrt{3} y}{2}} \\
& r_{12}(x, y) \\
& =R \sqrt{1-\sqrt{x^{2}+y^{2}} \cos \left(\arctan \left(\frac{y}{x}\right)\right)} \\
& =R \sqrt{1-x}
\end{aligned}
$$

Note that this can be simplified using the identities

$$
\begin{aligned}
\sin \left(\frac{\pi}{6}-\arctan \left(\frac{y}{x}\right)\right)=\frac{x-\sqrt{3} y}{2 \sqrt{x^{2}+y^{2}}} \\
\cos \left(\frac{\pi}{6}-\arctan \left(\frac{y}{x}\right)\right)=\frac{y+\sqrt{3} x}{2 \sqrt{x^{2}+y^{2}}} \\
r_{1}(x, y)=R \sqrt{\frac{1}{3}\left(1-\sqrt{x^{2}+y^{2}} \cos \left(\frac{\pi}{6}+\arctan \left(\frac{y}{x}\right)\right)\right)} \\
=R \sqrt{\frac{1}{3}\left(1+\frac{1}{2}(y-\sqrt{3} x)\right)} \\
r_{2}(x, y)=R \sqrt{\frac{1}{3}\left(1-\sqrt{x^{2}+y^{2}} \cos \left(\frac{\pi}{6}-\arctan \left(\frac{y}{x}\right)\right)\right)} \\
=R \sqrt{\frac{1}{3}\left(1-\frac{1}{2}(y+\sqrt{3} x)\right)}
\end{aligned}
$$

$$
\begin{aligned}
r_{3}(x, y) & =R \sqrt{\frac{1}{3}\left(1+\sqrt{x^{2}+y^{2}} \cos \left(\arctan \left(\frac{y}{x}\right)\right)\right)} \\
& =R \sqrt{\frac{1}{3}(1+x)}
\end{aligned}
$$

The above Eq. (A2) for the Y-string potential shows that it depends only on $\left(x^{2}+y^{2}\right)$. Converted into permutationadapted hyperspherical coordinates, this explicitly shows that $V_{Y}$ depends only on the hyperangle $\alpha$, and not on the hyperangle $\phi$, i.e., that the Y-string potential has an $O(2)$ dynamical symmetry, which is not shared by the $\Delta$-string potential $[16,18]$. This fact puts these two potentials into two distinct universality classes, in the sense of phase transitions in statistical mechanics, meaning that one cannot change one into another without a discontinuity in at least one variable [16].

We need the formulae for the Y- and $\Delta$-string potentials in the isosceles and right-angled configurations in terms of $(x, y)$ coordinates. We can choose one of three permutations; we shall use the "simplest" parametrization: (1) isosceles $y=0$; (2) right-angled triangles $x=-\frac{1}{2}$.

Therefore, the isosceles potentials are

$$
\begin{aligned}
& V_{\Delta}(x, y=0)=\sigma_{\Delta} R\left(\sqrt{1-x}+2 \sqrt{\frac{x}{2}+1}\right) \\
& V_{Y}(x, y=0)=\sigma_{Y} R \frac{\sqrt{\sqrt{1-x^{2}}+1}}{\sqrt{2}}
\end{aligned}
$$

and the right-angled triangles ones are

$$
\begin{aligned}
V_{\Delta}\left(x=-\frac{1}{2}, y\right)= & \sigma_{\Delta} R\left(\sqrt{\frac{1}{2}(-\sqrt{3} y-0.5)+1}\right. \\
& \left.+\sqrt{\frac{1}{2}(\sqrt{3} y-0.5)+1}+\sqrt{\frac{3}{2}}\right) \\
V_{Y}\left(x=-\frac{1}{2}, y\right)= & \sigma_{Y} R \sqrt{\frac{1+\sqrt{0.75-y^{2}}}{2}}
\end{aligned}
$$



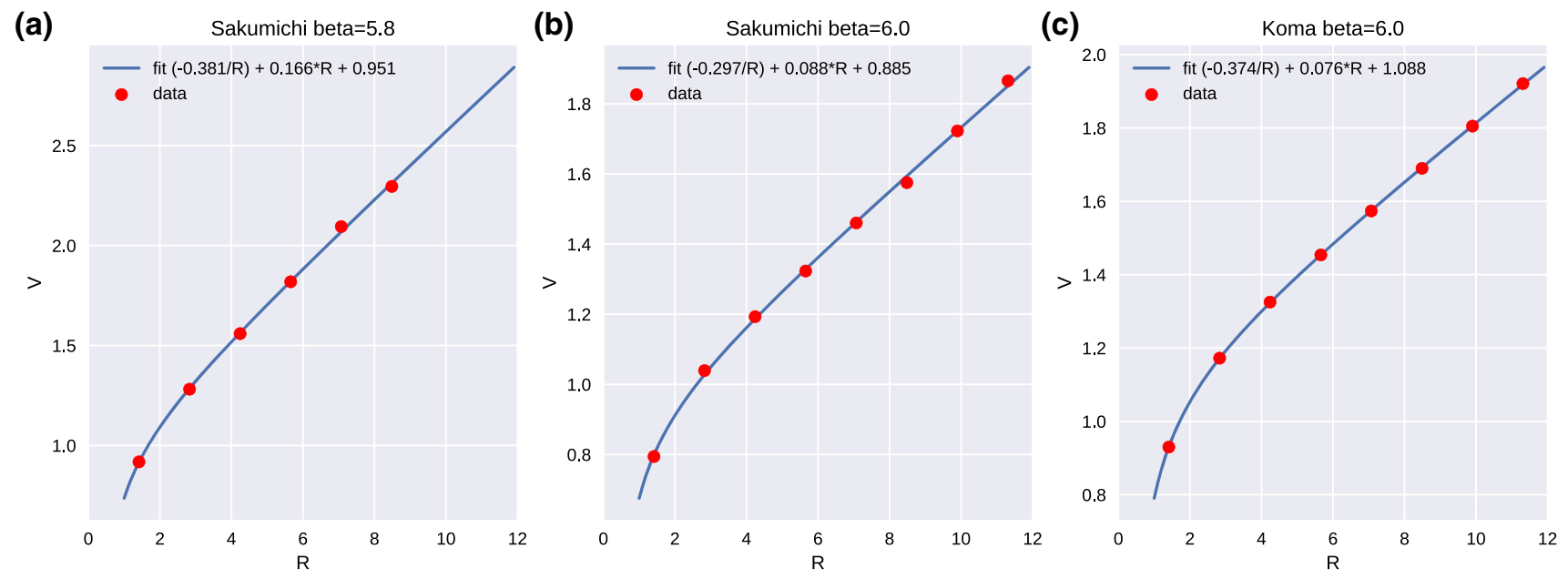

Fig. 5 Fit (blue solid line) to lattice data for the potential energy (red points) in the equilateral triangles configuration as a function of hyperradius $R$, using the Coulomb+linear Ansatz at a $\beta=5.8$ Ref. [1]; $\mathbf{b} \beta=6.0$ Ref. [1]; c $\beta=6.0$ Ref. [2]

Table 1 Our and Sakumichi and Suganuma's (from Table II in (1) fitted constants in the 3-quark potential for equilateral triangles: the Coulomb coefficient $A=3 A_{3 Q}$, the string tension $B$, and the constant
$C=C_{3 Q}$. Error bars are in parentheses - note the substantial difference in the error bars of our fitted values and those in Ref. [1]

\begin{tabular}{lllllll}
\hline Name & $A_{\text {fit }}$ & $B_{\text {fit }}$ & $C_{\text {fit }}$ & $A_{\text {Sak }}$ & $B_{\text {Sak }}$ & $C_{\text {Sak }}$ \\
\hline Sak $\beta=5.8$ & $0.381(77)$ & $0.166(6)$ & $0.951(53)$ & $0.357(9)$ & $0.168(2)$ & $0.93(1)$ \\
Sak $\beta=6.0$ & $0.297(42)$ & $0.088(2)$ & $0.884(26)$ & $0.363(9)$ & $0.081(2)$ & $0.936(9)$ \\
\hline
\end{tabular}

Figure 4 shows the $\Delta$ and Y-string potentials in the rightangled and the isosceles triangle configurations. Note that the difference is generally small (only about $2 \%$ in the isosceles right-angled configuration (Fig. 4a), except near the endpoint $y=-1$ (where the two-body collision singularity resides) where it grows to $50 \%$ (i.e. the $l_{\Delta}=2 l_{Y}$ is twice the Y-string length).

\section{Appendix B: Fitting the lattice data with the Coulomb+ Linear Ansatz}

We used the fixed equilateral triangle geometry with multiple sizes to fit the three free couplings A, B, C, see Table 1, to the data, see Fig. 5.

Note that the error bars of the Coulomb (A) and the constant term (C) are substantial: ranging from $21 \%$ for $\mathrm{A}$, and 5 $\%$ for $\mathrm{C}$, to only $2.4 \%$ for the string tension $\mathrm{B}$. This suggests that the (total) error bars in Ref. [10] were sometimes significantly underestimated, particularly in the Coulomb coupling. To be fair, we note that Koma and Koma [11] had also found a $26 \%$ variation of the Coulomb coupling over various spatial configurations. This suggests that the issue of Coulomb coupling leaves definite space for improvements, such as inclusion of as yet unknown generalizations of the so- called Lüscher term [30] in three-quark configurations. ${ }^{5} \mathrm{We}$ close with the (obvious) comment that such $1 / R$ corrections are immaterial for confinement potential.

\section{References}

1. S. Mandelstam, Phys. Lett. B 53, 476-478 (1975)

2. G. 't Hooft, Nucl. Phys. B 79, 276 (1974)

3. Y. Nambu, Phys. Rev. D 10, 4262 (1974)

4. S. Mandelstam, Phys. Rep. 23, 245-249 (1976)

5. R. Sommer, J. Wosiek, Phys. Lett. B 149, 497 (1984)

6. R. Sommer, J. Wosiek, Nucl. Phys. B 267, 531 (1986)

7. J. Flower, Nucl. Phys. B 289, 484 (1987)

8. H. Thacker, E. Eichten, J. Sexton, Nucl. Phys. B Proc. Suppl. 4, 234 (1988)

9. T.T. Takahashi, H. Suganuma, Y. Nemoto, H. Matsufuru, Phys. Rev. D 65, 114509 (2002)

10. N. Sakumichi, H. Suganuma, Phys. Rev. D 92(3), 034511 (2015)

11. Y. Koma, M. Koma, Phys. Rev. D 95(9), 094513 (2017)

12. J. Leech, M. Šuvakov, V. Dmitrašinović, Act. Phys. Pol. Suppl. B 11, 435 (2018)

13. J. Leech, M. Šuvakov, V. Dmitrašinović, Act. Phys. Pol. Suppl. B 14, 215-220 (2021)

14. V.G. Bornyakov et al. [DIK Collaboration], Phys. Rev. D 70, $054506(2004)$

15. F. Bissey, F.-G. Cao, A.R. Kitson, A.I. Signal, D.B. Leinweber, B.G. Lasscock, A.G. Williams, Phys. Rev. D 76, 114512 (2007)

\footnotetext{
${ }^{5}$ For one proposal, see Ref. [17].
} 
16. V. Dmitrašinović, T. Sato, M. Šuvakov, Phys. Rev. D 80, 054501 (2009)

17. P. de Forcrand, O. Jahn, Nucl. Phys. A 755, 475 (2005). arXiv:hep-ph/0502039

18. M. Šuvakov, V. Dmitrašinović, Phys. Rev. E 83, 056603 (2011)

19. H.J. Rothe, Lattice Gauge Theories: An Introduction, 3rd edn. World Scientific Lecture Notes in Physics, vol. 74 (2005)

20. J. Smit, Introduction to Quantum Fields on a Lattice- 'a robust mate' (Cambridge University Press, Cambridge, 2003)

21. C. Gattringer, C.B. Lang, Quantum Chromodynamics on the Lattice-An Introductory Presentation. Lecture Notes in Physics, vol. 788 (Springer, Heidelberg, 2010)

22. Interpretation of Lattice QCD Three-Quark Potential as a Flux Tube, or Hadronic String, talk delivered by V. Dmitrašinović, at the workshop "New Frontiers in QCD 2018-Confinement, Phase Transition, Hadrons, and Hadron Interactions-(NFQCD18), at the Yukawa Institute for Theoretical Physics, Kyoto University, Kyoto (22nd June 2018) (unpublished)
23. See the Wikipedia article at web site https://en.wikipedia.org/wiki/ Triangle_center

24. See the web site https://suvakov.github.io/3q/ for interactive analysis and https://github.com/suvakov/3q for the original data

25. I. Salom, V. Dmitrašinović, Nucl. Phys. B 920, 521 (2017)

26. V. Dmitrašinović, I. Salom, Phys. Rev. D 97, 094011 (2018)

27. I. Salom, V. Dmitrašinović, Act. Phys. Pol. Suppl. B 14, 121-126 (2021)

28. C. Ay, J.M. Richard, J.H. Rubinstein, Phys. Lett. B 674, 227 (2009). arXiv:0901.3022 [math-ph]

29. V. Dmitrašinović, Phys. Lett. B 499, 135-140 (2001). arXiv:hep-ph/0101007 [hep-ph]

30. M. Lüscher, K. Symanzik, P. Weisz, Nucl. Phys. B 173, 365 (1980) 